Case Report

\title{
Concrescence of permanent maxillary second and third molars: case report of non-surgical root canal treatment
}

\author{
Denise Foran ${ }^{1)}$, Takashi Komabayashi²) and Louis M. Lin ${ }^{1)}$ \\ 1)Department of Endodontics, New York University College of Dentistry, New York, NY, USA \\ 2)Department of Endodontics, Texas A\&M Health Science Center Baylor College of Dentistry, \\ Dallas, TX, USA
}

(Received 24 October 2011 and accepted 23 January 2012)

\begin{abstract}
Concrescence is a rare developmental anomaly with an overall incidence of $0.8 \%$ in the permanent dentition. While many case reports describe the treatment of concrescence with extraction, there are few reports of non-surgical root canal treatment (NSRCT), due to the atypical root form, canal morphology, and technical difficulties involved in concrescence. This unique case report describes a technical modification of NSRCT that can retain joined posterior maxillary teeth to maintain natural posterior occlusion without surgical intervention or dental implants, thereby avoiding the risk of damage to a large portion of the alveolar bone near the maxillary sinus. (J Oral Sci 54, 133-136, 2012)
\end{abstract}

Keywords: concrescence; fused teeth; root canal treatment; tooth abnormalities/complications.

\section{Introduction}

Concrescence is a rare dental anomaly defined as the cemental union of two adjacent teeth (1). These joined teeth have independent pulp chambers and root canal systems. Concrescence has been reported in extraction cases with an incidence of $0.2-3.7 \%$ in the primary dentition and $0.8 \%$ in the permanent dentition (2). Nonsurgical root canal treatment (NSRCT) is a challenge in such cases due to atypical root anatomy, unpredictable

Correspondence to Dr. Denise Foran, Department of Endodontics, New York University College of Dentistry, 345 E. 24th Street, New York, NY 10010, USA

Tel: +1-917-589-2727

E-mail: df67@nyu.edu root canal systems, risk of missing canals, and perforations. The aim of this case report is to present a successful NSRCT of a right maxillary second and third molar joined by a cemental union.

\section{Case Report}

A 35-year-old female presented to private practice limited to endodontics for evaluation of dental pain. The patient's chief complaint was lingering sensitivity to cold in the maxillary right quadrant. Her medical history was unremarkable. She had no drug allergies, medications or any cardiac and/or joint diseases requiring antibiotic prophylaxis. Her dental history included routine dental care, low caries risk and good oral hygiene. The extra oral exam and perioral soft tissue exam were within normal limits. Her temporomandibular joint function was normal without any deviation upon opening or discomfort upon palpation, and the intraoral hard examination revealed a dense cortical plate in the posterior maxilla; the soft tissues had no signs or symptoms of pathology. Clinically, tooth \#18 had an occlusal composite filling present and tooth \#17 has an Intermediate Restorative Material (IRM, Dentsply Caulk, Milford, DE, USA). (Figs. 1A and 1B) Interestingly, the crowns and roots of these two teeth were joined and both teeth exhibited signs and symptoms of patient's chief complaint. Clinical examination and intraoral photographs did not reveal any cracks or fractures, and there was no discomfort upon percussion, palpation or bite stick testing. Her chief complaint was reproducible when teeth \#17 and \#18 were tested with EndoIce (Coltène Whaledent, Cuyahoga Falls, OH, USA). Radiographic examination revealed intact periodontal ligament space around teeth 

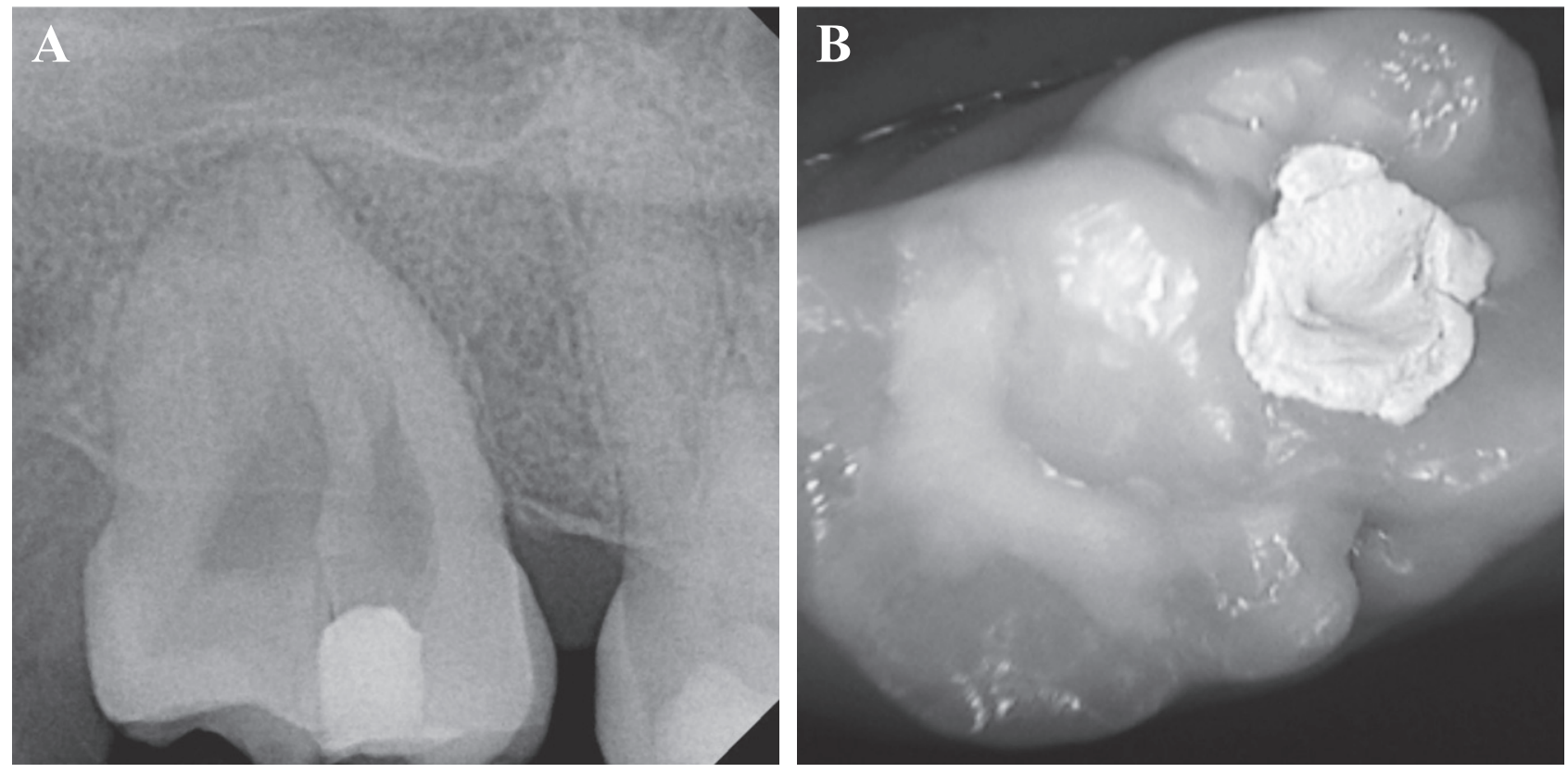

Fig. 1 Preoperative. A. Radiograph of teeth \#18 and \#17, B. Intraoral occlusal view of teeth \#18 (Composite resin filling) and \#17 (IRM restoration).
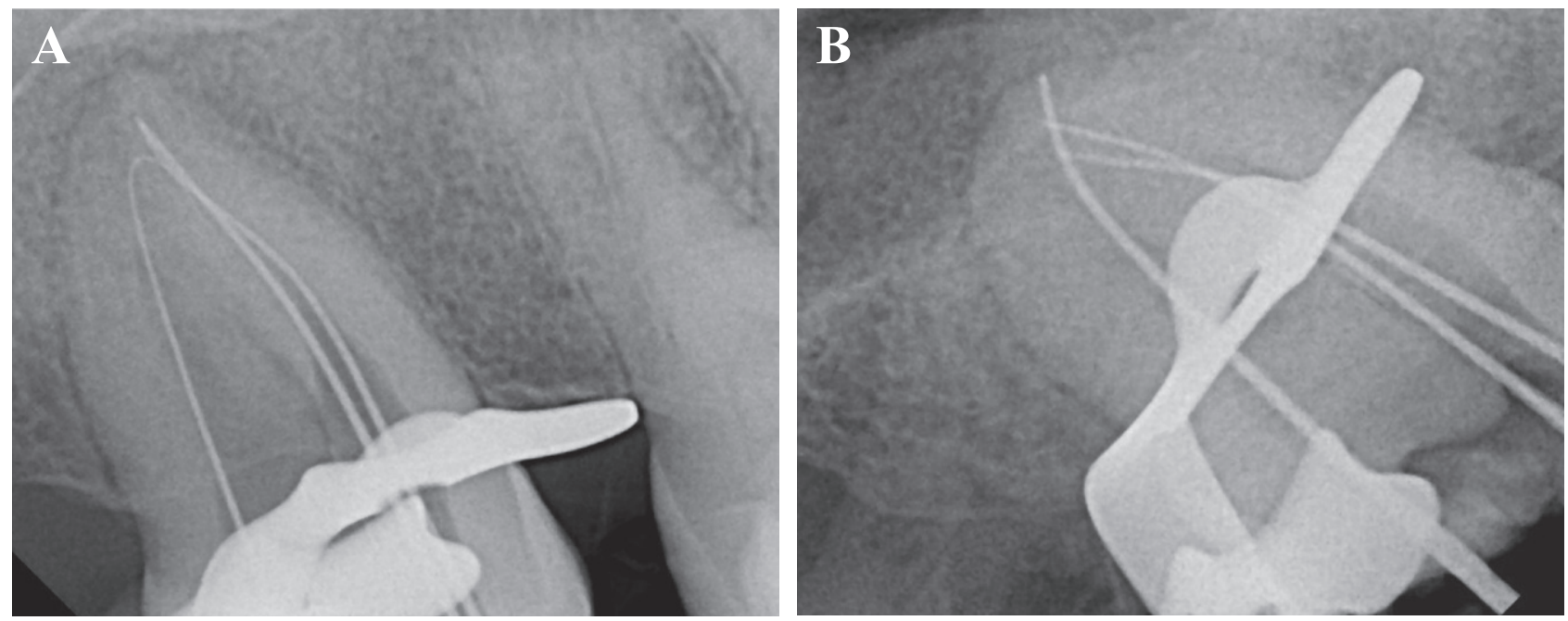

Fig. 2 Measurement films. A. Straight on, B. Mesial shift angulation.

\#17 and \#18 and no evidence of periapical pathosis. The endodontic diagnosis for this patient was determined to be irreversible pulpitis with normal periapex. Treatment options were discussed with patient including NSRCT or extraction. Considering risks and benefits of both procedures, an agreement was made to proceed with NSRCT.

The endodontic retreatment for this tooth was started the day of the evaluation to alleviate patient's discomfort. Local maxillary infiltration of $3.6 \mathrm{ml}$ of $2 \%$ lidocaine with $0.072 \mathrm{mg}$ of epinephrine was administered (SAFKO Dental Supply Co., Buffalo Grove, IL, USA). Although the patient did not have limited opening, a prominent coronoid process made rubber dam placement challenging. Dental clamp and dental dam modifications were necessary to achieve the appropriate isolation; they needed to be placed with the patient at $50 \%$ of her maximum opening to attain optimal isolation from the oral cavity. Access cavity preparation was challenging due to multiple factors besides the intentional limited opening; these factors included mandibular anatomy, atypical root form and unusual canal morphology. The use of magnification, illumination, and methylene blue staining were critical in proper debridement, canal identification, and reducing possibility of missed canals. Working lengths were determined with the use of an apex locator (Root ZX, Morita, Tokyo, Japan), and measurement films taken at multiple angulations to visualize canal configurations and apical termini (Figs. 2A and 2B). 

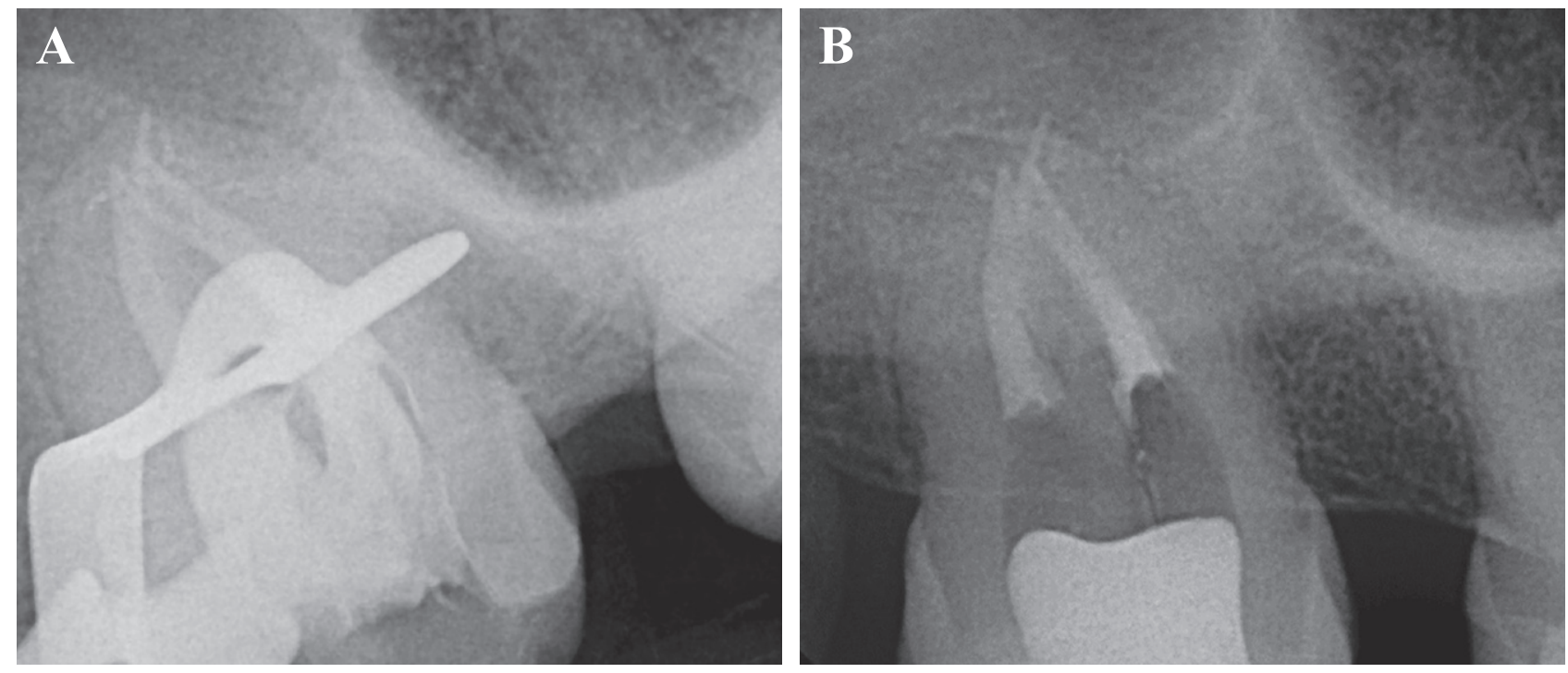

Fig. 3 A. Master cone film after completion of lateral condensation, B. Final film temporized with cotton pellet and Fuji TRIAGE.

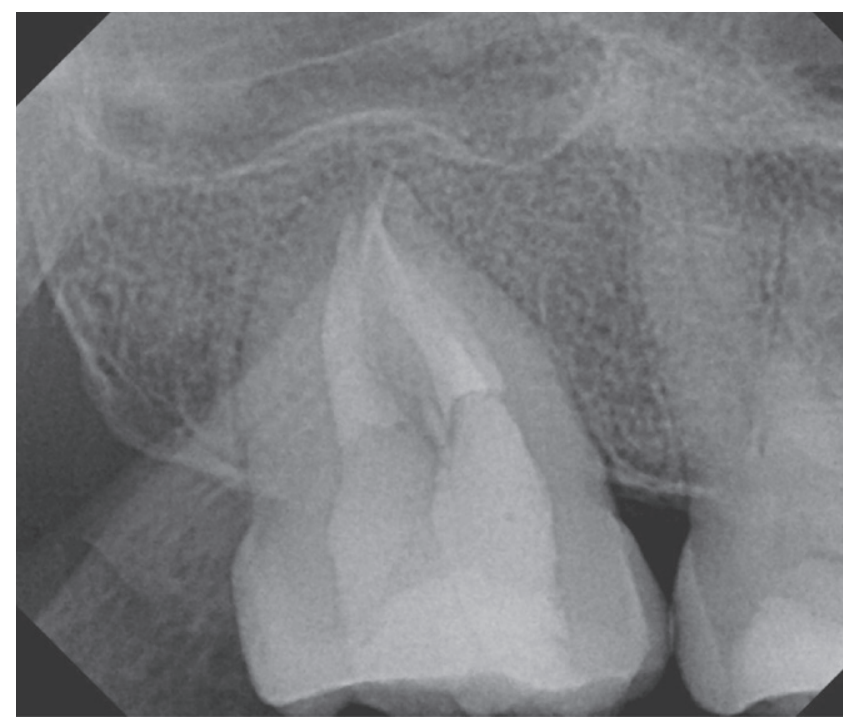

Fig. 4 Eighteen-month follow-up radiograph with permanent composite restoration.

Debridement was accomplished using hand instrumentation with K-type and Hedstrom files (Dentsply-Maillefer, Tulsa, OK, USA), and rotary instrumentation using Profile (Dentsply-Maillefer). Rotary files were not taken to measurement due to canal configuration and risk of instrument separation. Copious irrigation of $5.25 \%$ sodium hypochlorite solution was used during cleaning and shaping process followed by a final rinse of $17 \%$ ethylenediaminetetraacetic acid (EDTA) to remove the smear layer. The teeth were then temporized with a cotton pellet and Fuji TRIAGE (GC America, Alsip, IL, USA).

The patient presented one week later for follow up. Patient was asymptomatic and chief complaint had been resolved. Administration of local anesthesia and rubber dam placement technique was used explained during the first visit. Gutta percha size medium (DiaDent Group International, Burnaby, BC, Canada) was selected to obtain attain appropriate apical fit and tug back. After all master gutta percha points were fit to measurement; the canals were dried with medium paper points (DiaDent Group International). After canals were dried thoroughly, all canals were obturated using lateral condensation with a nickel titanium endodontic spreader (Hu-Friedy, Chicago, IL, USA) and Roth Grossman's root canal sealer (Pearson Dental, Sylmar, CA, USA). This techniques aim was to achieve a three-dimensional fill and to eliminate the risk of not seating all points to their proper apical position in the event there was an undetectable root communication. After obturation was complete, excess gutta percha was removed from the chamber using a heated posterior endodontic plugger (Hu-Friedy). The teeth were again temporized with cotton pellet and Fuji TRIAGE (GC Co., Tokyo, Japan) was used as a temporary seal of the coronal access (Figs. 3A and 3B). The patient was referred to her general dentist for permanent restoration and continuation of care.

The patient was recalled for a 15-month follow-up to treatment, and the outcome was excellent. The patient has remained asymptomatic since the completion of NSRCT, and radiographic exam remained free of periapical pathosis. The permanent restoration placed by the general was intact without any evidence of leakage. (Fig. 4)

\section{Discussion}

Due to the rare incidence of concrescence in the permanent dentition, this case is important to document 
because it demonstrates a successful conservative treatment option that can be used to retain teeth in adults without surgical intervention. It is often difficult to diagnose concrescence radiographically since the teeth are joined by cementum only, have independent pulp chambers and root canal systems. This is a plausible explanation as to why most of these cases are diagnosed and reported post extraction (3).

The successful results of this rare case can be attributed to multiple factors including proper diagnosis and treatment modifications. Although the root canal systems of such teeth are separate, both teeth independently exhibited signs and symptoms of a lingering response to thermal testing suggesting irreversible pulpitis and necessitating endodontic therapy. Unlike previous case reports this concrescence did not involve supernumerary teeth and did not involve mandibular teeth (4-7). The loss of permanent maxillary second and third molars that are in a stable occlusion may have a more profound negative impact on the patient's masticatory function than the loss of supernumeraries and third molars alone. The extracted concrescent teeth in primary dentition have permanent successors, while extracted permanent dentition does not have successors. The extractions of such teeth in the permanent dentition may pose more risks than benefits and would be better managed with NSRCT despite the challenges the clinician may encounter. Although extracting these pulpally compromised teeth is a viable option, the loss of the alveolar plate or sinus perforation may be consequential. This case is clinically relevant because the extraction of teeth \#18 and \#17 posed a high risk of damage to a large portion of the alveolar bone and possible sinus perforation. Long-term outcome studies in concrescence will be helpful for further clinical research, and the use of Cone Beam Computed Tomography (CBCT) would be a powerful adjunct in such cases by capturing threedimensional images. This enhanced imaging will better equip the clinician to visualize external root morphology and internal root canal anatomy in concrescence cases and would provide more diagnostic information than conventional two-dimensional digital radiography $(5,8)$. This technology was not available at the clinical facility and patient could not afford to have the imaging done at another location. The significance of this rare clinical case is that NSRCT can retain posterior concrescent teeth in order to maintain a natural posterior occlusion without surgical intervention or dental implants.

\section{Acknowledgments}

The authors thank Ms. Jeanne Santa Cruz (Texas A\&M Health Science Center Baylor College of Dentistry) for the critical editing of this paper.

\section{References}

1. American Association of Endodontists (2003) Glossary of endodontic terms. Chicago.

2. Law L, Fishelberg G, Skribner JE, Lin LM (1994) Endodontic treatment of mandibular molars with concrescence. J Endod 20, 562-564.

3. Gernhofer KJ (2009) Concrescence of a maxillary second and third molar. J Calif Dent Assoc 37, 479-481.

4. Friedman S, Stabholz A, Rotstein I (1986) Endodontic management of molars with developmental anomalies. Int Endod J 19, 267-276.

5. Song CK, Chang HS, Min KS (2010) Endodontic management of supernumerary tooth fused with maxillary first molar by using cone-beam computed tomography. J Endod 36, 1901-1904.

6. Goldberg JM, Gross M, Rankow H (1985) Endodontic therapy involving fused mandibular second and third molars. J Endod 11, 346-347.

7. Kremeier K, Pontius O, Klaiber B, Hülsmann M (2007) Nonsurgical endodontic management of a double tooth: a case report. Int Endod 40, 908-915.

8. Ballal S, Sachdeva GS, Kandaswamy D (2007) Endodontic management of a fused mandibular second molar and paramolar with the aid of spiral computed tomography: a case report. J Endod 33, 1247-1251. 